\title{
A Network Reliability Game
}

\author{
Patrick Maillé \\ IMT Atlantique \\ Rennes, France \\ patrick.maille@imt.fr
}

\author{
Bruno Tuffin \\ Inria, Univ Rennes, CNRS, IRISA \\ Rennes, France \\ bruno.tuffin@inria.fr
}

November 29, 2018

\begin{abstract}
In an ad hoc network, accessing a point depends on the participation of other, intermediate, nodes. Each node behaving selfishly, we end up with a non-cooperative game where each node incurs a cost for providing a reliable connection but whose success depends not only on its own reliability investment but also on the investment of nodes which can be on a path to the access point. Our purpose here is to formally define and analyze such a game: existence of an equilibrium output, comparison with the optimal cooperative case, etc.
\end{abstract}

\section{Introduction: problem definition}

We consider a topology with nodes wishing to be connected to a network access point. If from a node there is no direct connection to the access point, the connection has to be routed through other nodes. The feasibility/success/quality you experience as a node therefore depends on the quality you provide yourself, but also on the participation of peers to the network.

This type of problem has many applications. Typical, but non-exhaustive, ones are:

- An ad hoc network [9], which is a network without any fixed infrastructure where nodes serve as relays for their neighbors. Such an organization presents the advantages of being decentralized, and of incurring no deployment cost since relying on the collaboration and willingness to participate of nodes. There are many practical applications of those networks: Army tactical ad how networks for a fast deployment and operation in a war context when no fix/wireless network exists, smart phone ad hoc networks not requiring the traditional wireless carriers, vehicular ad hoc networks for autonomous vehicles, sensor networks, etc. Investment/participation of nodes is a key issue for the success in these applications. 
- Security/reliability issues: nodes in a network can be subject to (stochastic) failures reducing the path possibilities to the target. There may also be attacks on some nodes, rendering paths to the target through infected nodes not practicable. Investing on security procedures limits the infection risks but incurs a cost; this trade-off and the impact on the whole system in the case of selfish nodes requires attention.

The natural modeling framework is that of non-cooperative game theory $[2,6]$. In the literature, security and related investment games have been studied quite a lot; see for instance $[4,5]$ and the references therein. Security and free riding have been studied in [10]. The closest paper to ours is [3], where a general framework for security investment is provided, with functional interactions between nodes. Our model fits this framework in general, but we focus here on something more specific, and our utility functions are different, hence the results in [3] need to be adapted.

Our contribution is the following: we design a graph model with nodes as players and links corresponding to possible direct connections between nodes. Each node is subject to failures and has to set up its reliability for a given cost. On the other hand, each node is interested in the existence (in probability) of a path to the target access node, meaning that its service quality also depends on the others' choices. Our specificity in this game-theoretic context is therefore the use of the so-called static reliability analysis which computes the probably of such a path. Numerous methods exist for this NP-hard problem, efficient Monte Carlo simulation being applied in the case of large networks $[1,8]$. We show how this problem can be analyzed, with specific results.

The remaining of the paper is organized as follows. Section 2 describes the formal mathematical model we are going to analyze and the goals of the paper. Section 3 presents two illustrative examples, helpful to grasp the stakes and difficulties at hand, as well as some results on the Price of Anarchy, measuring the loss of efficiency due to selfishness. The general results on the output of the game are presented in Section 4, and Section 5 concludes the paper by giving directions of future research.

\section{Model}

\section{$2.1 \quad$ Graph model}

We consider an undirected connected graph $\mathcal{G}=(\mathcal{N}, \mathcal{L})$ where $\mathcal{N}=\{0,1, \ldots, n\}$ is the set of nodes, and $\mathcal{L}$ is the set of links/possible connections between nodes. Node numbered 0 is the access node. Links are assumed to always work, but nodes 1 to $n$ are subject to (independent) failures, due to vulnerabilities, attacks, etc.. The access node is assumed perfect. Let $r_{i}$ be the probability to be up for Node $i$, and $q_{i}=1-r_{i}$. A configuration of the graph is given by vector $X=\left(X_{1}, \ldots, X_{n}\right)$ where $X_{i}=1$ if $i$ is up and 0 if it is down. Retaining only the set $\mathcal{N}^{\prime}$ of "up" nodes, we obtain a random partial graph $\mathcal{G}^{\prime}=\left(\mathcal{N}^{\prime}, \mathcal{L}\right)$.

Node $i$ is interested in the probability $R_{i}$ that in $\mathcal{G}^{\prime}$ there is a path connecting $i$ to 
0 . This type of problem has been extensively studied in the literature [1, 8]; basically,

$$
R_{i}=\sum_{x \in\{0,1\}^{n}} \Psi_{i}(x) \mathbb{P}[X=x]=\sum_{x \in\{0,1\}^{n}} \Psi_{i}(x) \prod_{j=1}^{n}\left(r_{j} x_{j}+q_{j}\left(1-x_{j}\right)\right)
$$

where $\Psi_{i}(x)$ is 0 if there no path in the subgraph $\mathcal{G}^{\prime}$ corresponding to configuration $x$ (a vector $\left(x_{1}, \ldots, x_{n}\right)$ where $x_{i}=1$ means Node $i$ is up), and 1 if there is at least one such path. It is easy to compute analytically for small topologies, as a finite sum, but it is in general an NP-hard problem for which approximation methods, among which efficient simulation algorithms, must be used for large topologies. See $[1,8]$ for a general description of the methodologies. Since it is not the purpose of the present paper, we assume that how to get a sufficiently accurate estimation is known.

\subsection{Utility functions}

We assume that Node $i$ has a valuation $f_{i}\left(R_{i}\right)$ for the service, depending on the probability to get a connection to the access point. Each function $f_{i}$ is assumed increasing: the larger the quality/reliability, the more it is appreciated, and $f_{i}$ is also assumed concave, a usual and reasonable assumption to express that getting a given reliability amount when you are at a low level is seen more valuable than when you are at a high level.

Node $i$ is also assumed to experience a cost $c_{i}\left(r_{i}\right)$ to maintain a level $r_{i}$ of reliability/security/quality of its own equipment. Function $c_{i}$ is assumed increasing too, and strictly convex, with $c_{i}(0)=0$ and $\lim _{r \rightarrow 1} c_{i}(r)=\infty$, again standard assumptions.

The utility $u_{i}$ of Node $i$ is then

$$
u_{i}\left(r_{1}, \ldots, r_{n}\right)=f_{i}\left(R_{i}\right)-c_{i}\left(r_{i}\right)
$$

where we recall that $R_{i}$ is a function of $r=\left(r_{1}, \ldots, r_{n}\right)$.

We end up with a non-cooperative game where each node $i$ plays with the level of investment resulting in (equivalent) reliability $r_{i}$, but his/her strategy impacts the valuation of other nodes.

\subsection{Questions to be answered}

From the definition of this problem, here are the questions we aim at answering:

- What is the outcome of the game? Does a Nash equilibrium exist? If yes, is it unique?

- How does the output compare with a cooperative situation? This is evaluated by computing the Price of Anarchy (PoA) measuring how the efficiency of a system degrades due to selfish behavior of its players as the ratio of optimal social utility divided by the worst social utility at a Nash equilibrium (the social utility being the sum of utilities over all players). 


\section{Examples}

\subsection{A simple example with non-trivial interactions among nodes}

Consider the topology described in Figure 1 where each node $i \in\{1, \ldots, 4\}$ wants to be connected to node 0 . We let $c_{i}(x)=\alpha \frac{x}{1-x}$ and $f_{i}(x)=x$, which verifies the

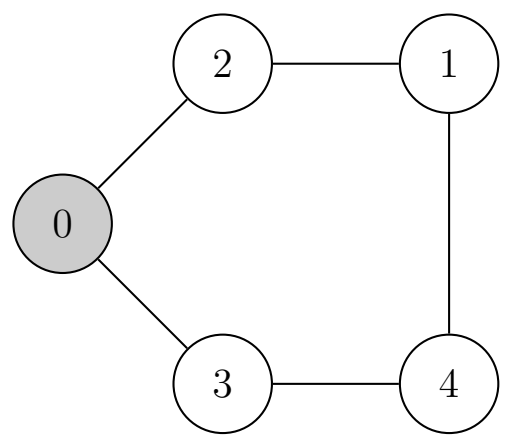

Figure 1: A simple topology

assumptions listed in the previous section.

Since Node $i$ is on all paths to Node 0 , reliability $R_{i}$ can be expressed as $R_{i}=r_{i} R_{i}^{\prime}$ where $R_{i}^{\prime}$ is the probability that there is a path from Node $i$ to Node 0 when $i$ is operational, and $R_{i}^{\prime}$ does not depend on $r_{i}$.

To compute the best response of Node $i$, we differentiate $u_{i}=r_{i} R_{i}^{\prime}-\alpha \frac{r_{i}}{1-r_{i}}$ with respect to $r_{i}$. It gives the optimal value

$$
r_{i}=\left[1-\sqrt{\alpha / R_{i}^{\prime}}\right]^{+}
$$

with $x^{+}=\max (x, 0)$.

For Nodes 2 and 3 at distance 1 of node 0 , hence with $R_{i}^{\prime}=1$, we then get $r_{i}=0$ if $\alpha \geq 1$ (that is, the investment cost is too high and the node prefers not to participate), and $1-\sqrt{\alpha}$ otherwise.

Let us look at Nodes 1 and 4 now. Observe that we have $R_{1}^{\prime}=r_{2}+\left(1-r_{2}\right) r_{3} r_{4}$ and $R_{4}^{\prime}=r_{3}+\left(1-r_{3}\right) r_{2} r_{1}$. The utility of one node depends on the reliability choice of the other, therefore we have a game between nodes 1 and 4 . Looking for the solution, we try to solve the system of equations

$$
\begin{aligned}
& \frac{\partial u_{1}}{\partial r_{1}}=r_{2}+\left(1-r_{2}\right) r_{3} r_{4}-\frac{\alpha}{\left(1-r_{1}\right)^{2}}=0 \\
& \frac{\partial u_{4}}{\partial r_{4}}=r_{3}+\left(1-r_{3}\right) r_{2} r_{1}-\frac{\alpha}{\left(1-r_{4}\right)^{2}}=0
\end{aligned}
$$




\begin{tabular}{c|c|c}
$\alpha$ & $r_{2}=r_{3}$ & $r_{1}=r_{4}$ \\
\hline 0.3 & 0.4522774425 & 0.2330400670 \\
0.1 & 0.6837722340 & 0.6517825785 \\
0.01 & 0.9 & 0.8990317600 \\
0.001 & 0.9683772234 & 0.9683460395 \\
0.0001 & 0.99 & 0.9899990048
\end{tabular}

Table 1: Some numerical results in terms of $\alpha$ for the example of Section 3.1

giving

$$
\begin{aligned}
& r_{1}=1-\sqrt{\frac{\alpha}{r_{2}+\left(1-r_{2}\right) r_{3} r_{4}}} \\
& r_{4}=1-\sqrt{\frac{\alpha}{r_{3}+\left(1-r_{3}\right) r_{2} r_{1}}} .
\end{aligned}
$$

Solving it is intractable in general, but we can easily get numerical results. Examples of solutions in terms of $\alpha$ are given in Table 1 . The lower the cost, the higher the reliability.

For this example with homogeneous utility valuations and costs, it is interesting to see that all $r_{i}$ are converging to 1 as $\alpha \rightarrow 0$ : the lower the investment cost, the better it is to participate because there is a gain at no cost.

But we can say more when $\alpha$ tends to 0 :

$$
r_{1}=r_{4}=1-\sqrt{\alpha} / \sqrt{(1-\sqrt{\alpha})\left(1+r_{1} \sqrt{\alpha}\right)}=1-\sqrt{\alpha}(1+o(1))
$$

where the $o(\cdot)$ is for $\alpha \rightarrow 0$. This yields $r_{1}=r_{2}+o(\sqrt{\alpha})$. Hence the $r_{i}$ are asymptotically equivalent, even in relative terms if we consider the difference with respect to 1 since $1-r_{1}=\sqrt{\alpha}+o(\sqrt{\alpha})=1-r_{2}+o\left(1-r_{2}\right)$, i.e., $\frac{1-r_{1}}{1-r_{2}}=1+o(1)$.

Finally, we can look at what would happen if the nodes were collaborating, that is, if they were trying to maximize the social utility defined as

$$
\sum_{i=1}^{4} u_{i}=r_{2}+r_{3}+r_{1}\left(r_{2}+\left(1-r_{2}\right) r_{3} r_{4}\right)+r_{4}\left(r_{3}+\left(1-r_{3}\right) r_{2} r_{1}\right)-\alpha \sum_{i=1}^{4} \frac{r_{i}}{1-r_{i}} .
$$

Getting the global optimum for $\left(r_{1}, \ldots, r_{4}\right) \in[0,1]^{4}$ seems intractable, but we can easily get numerical values again. Table 2 displays for the same values of $\alpha$ as in Table 1 the maximum value of $\sum_{i=1}^{4} u_{i}$, the corresponding $r_{i} \mathrm{~s}$, and the Price of Anarchy, that is that optimal value divided by the value of $\sum_{i=1}^{4} u_{i}$ at equilibrium. Note that the PoA is necessarily larger than 1. It is interesting to note that the Price of Anarchy is close to one. It also seems to converge to 1 as $\alpha \rightarrow 0$. In other words, selfishness of nodes does lead to negligible losses with respect to the cooperative case, particularly as $\alpha$ is close to 0 . 


\begin{tabular}{c|c|c|c}
$\alpha$ & Optimum $\left(r_{1}=r_{4}, r_{2}=r_{3}\right)$ & $\sum_{i=1}^{4} u_{i}$ & PoA \\
\hline 0.3 & $(0.3445638958,0.5265329288)$ & 0.4924406891 & 1.06014 \\
0.1 & $(0.6842015722,0.7383790483)$ & 1.670241284 & 1.021059 \\
0.01 & $(0.9027739526,0.90999676)$ & 3.208617313 & 1.000922 \\
0.001 & $(0.9687904071,0.969673)$ & 3.747335781 & 1.0000352 \\
0.0001 & $(0.9900470095,0.9900)$ & 3.920006440 & 1.000000122
\end{tabular}

Table 2: Social Optimum and Price of Anarchy in terms of $\alpha$ for the example of Section 3.1

\subsection{A simple example with unbounded Price of Anarchy}

We introduce here another simple example, where the interactions among nodes is not really a game (only one node affects the others), but the example illustrates what can happen in terms of the Price of Anarchy.

Let us consider the topology of Figure 2, with only Node 1 directly connected to the target node, and Nodes 2 to $n$ only connected to Node 1.

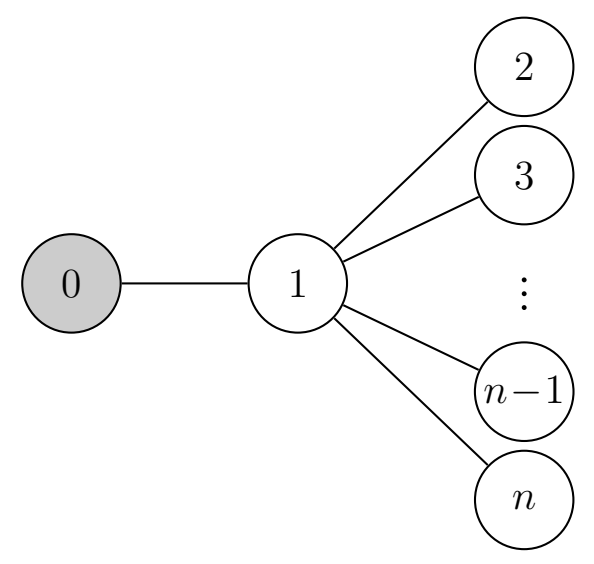

Figure 2: A specific topology with $n$ nodes

The Nash equilibrium is easy to compute, since the actions of Nodes 2 to $n$ only affect themselves and not Node 1. For Node 1, from (1) the Nash equilibrium (NE) strategy is simply

$$
r_{1}=[1-\sqrt{\alpha}]^{+}:=x^{\mathrm{NE}} .
$$

Then Nodes 2 to $n$ all see $R_{i}^{\prime}=r_{1}$, and therefore they all set, again from (1)

$$
r_{2}=\cdots=r_{n}=\left[1-\sqrt{\frac{\alpha}{[1-\sqrt{\alpha}]^{+}}}\right]^{+}:=y^{\mathrm{NE}} .
$$

Now, let us investigate the globally optimal choices. By symmetry, Nodes 2 to $n$ should set the same reliability level $y$, and if we denote by $x$ the reliability of Node 

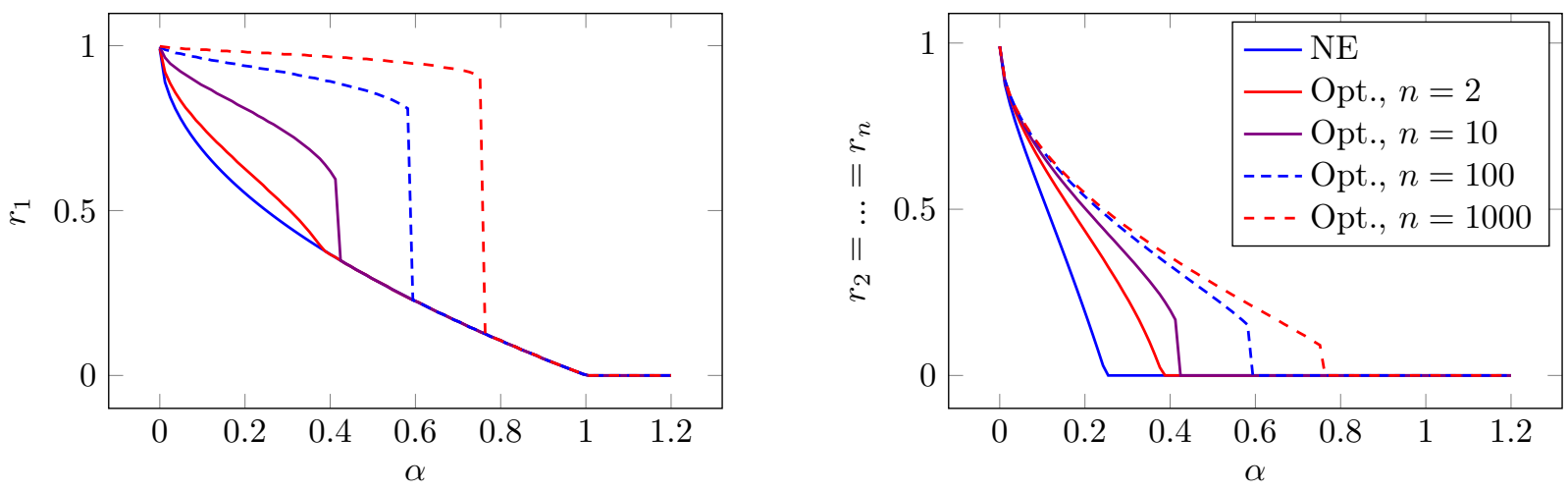

Figure 3: Equilibrium and globally optimal strategies for Node 1 (left), and for the other nodes (right), for the instance of Figure 2.

1 , the objective function is

$$
\sum_{i=1}^{n} u_{i}=x-\alpha \frac{x}{1-x}+(n-1)\left(x y-\alpha \frac{y}{1-y}\right),
$$

which gives $y=\left[1-\sqrt{\frac{\alpha}{x}}\right]^{+}$, and can be plugged into (2) to compute the optimal $x$ (we do this numerically to avoid cumbersome algebra). But we can already notice that the Price of Anarchy is unbounded in general: if we take $\alpha=0.49$, at equilibrium Node 1 selects $r_{i}=1-\sqrt{\alpha}=0.3$ which leads all the other nodes to select $r_{i}=0$, so the social utility is $u_{1}^{\mathrm{NE}}=0.09$. On the other hand, the optimal social utility is at least the one we would reach with $r_{1}=0.9$ and $r_{2}=r_{3}=\ldots=r_{n}=0.3$, which equals $-3.57+0.06 n$, hence the Price of Anarchy is at least $\frac{2}{3} n-40$, which increases linearly with $n$.

To treat our example for a more general set of parameters, Figure 3 shows the equilibrium and globally optimal strategies of Node 1 , and of Nodes 2 to $n$. The corresponding costs are computed according to (2), and are compared through the Price of Anarchy, plotted in Figure 4. As in the previous example, we observe that the Price of Anarchy tends to 1 when $\alpha$ tends to 0 (if the cost of reliability is null, all nodes should select $r_{i}$ close to 1 , both at equilibrium and at the socially optimal outcome).

\section{Analytical results}

While the previous section was dealing with instructive examples, we provide here general results towards the analysis of Nash equilibria and the resulting outcome.

A first result, observed on the examples in the previous section, is about the nodes directly connected to the access point (Node 0):

Proposition 1. For nodes at distance 1 from Node 0 , the decision does not depend on other nodes. 


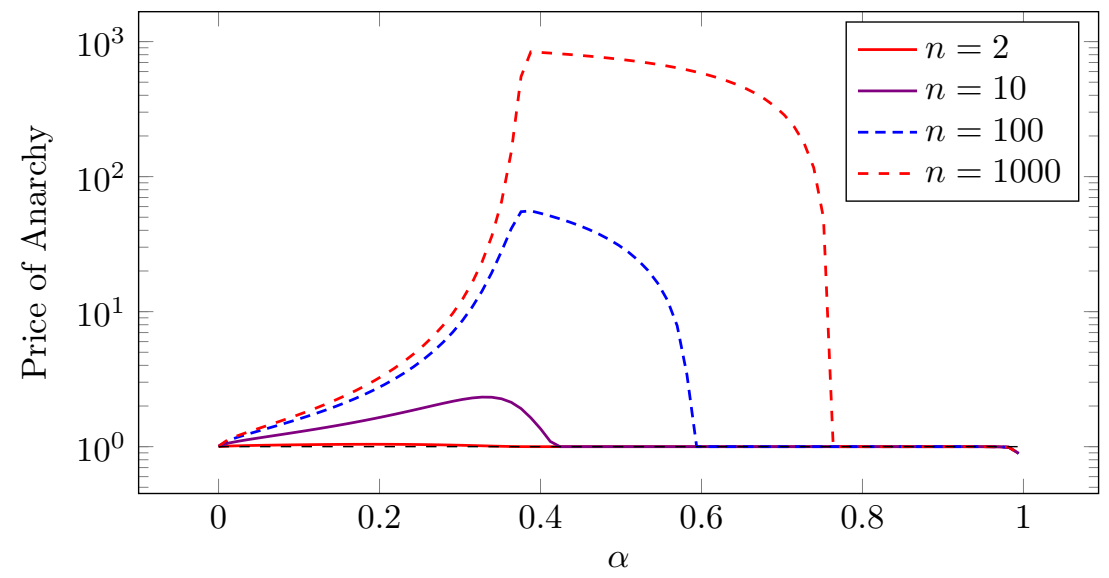

Figure 4: Price of Anarchy for the instance of Figure 2.

Proof. It comes from the fact that for such nodes, $R_{i}^{\prime}=1$ because there is a direct link between $i$ and 0 .

We now get interested in the Nash equilibria of the game. Since Nash equilibria are fixed-points of best-response correspondences, we first provide results on bestresponses.

Proposition 2. Given the assumptions of concavity of $f_{i}$ and strict convexity of $c_{i}$, Node $i$ has a unique best response in $[0,1)$.

Proof. Note that $R_{i}$ is a linear function of $r_{i}$ (actually $R_{i}=r_{i} R_{i}^{\prime}$ with $R_{i}^{\prime}$ the reliability of the random subgraph where both nodes 0 and $i$ are perfect, and $R_{i}^{\prime}$ does not depend on $\left.r_{i}\right)$. As a consequence $u_{i}\left(r_{1}, \ldots, r_{n}\right)$ is strictly concave in $r_{i}$, hence the result.

Proposition 3. The game has at least one Nash Equilibrium.

Proof. It is a direct consequence of Rosen's theorem with concave utility functions, with action sets on a compact, here $[0,1]$ for each player.

This is similar to what was shown in [3], something expected. Regarding the equilibrium uniqueness, we can apply existing results to express a sufficient condition, but that condition is difficult to verify in practice:

Proposition 4. Let $U(r)$ be the Jacobian matrix $U(x)=\left(\frac{\partial u_{i}(r)}{\partial r_{j} \partial r_{i}}\right)_{1 \leq i, j \leq n}$. Let $U^{t}(r)$ be the transpose matrix of $U(r)$. If the symmetric matrix $U(r)+U^{t}(r)$ is negative definite (that is, for all $r \in[0,1]^{n}$ we have $y^{t}\left(U(r)+U^{t}(r)\right) y<0 \forall y \neq 0$ ) the Nash equilibrium is unique.

Proof. It is a sufficient condition of Rosen's uniqueness condition [7]. 


\section{Conclusions}

In this paper, we have introduced a specific model for the interactions among participants (nodes) in an ad-hoc network in terms of reliability investments, when all nodes want a reliable access to a given point. When nodes are selfish, the non-cooperative game can be difficult to analyze, but we have proved that, under reasonable conditions, it always has an equilibrium. We have highlighted the contrast with globally optimized decisions, for which the investments of nodes close to the sink are higher than in the non-cooperative setting, due to the positive externality they create. We have shown that the loss of efficiency due to user selfishness, measured through the Price of Anarchy, can be arbitrarily large in general, but is in practice small for many settings (in particular, when reliability is cheap). This suggests some careful consideration is needed before deciding whether some coordination should be enforced.

\section{References}

[1] H. Cancela, M. El Khadiri, and G. Rubino. Rare events analysis by Monte Carlo techniques in static models. In G. Rubino and B. Tuffin, editors, Rare Event Simulation using Monte Carlo Methods, pages 145-170. John Wiley \& Sons, 2009.

[2] D. Fudenberg and J. Tirole. Game Theory. MIT Press, Cambridge, Massachusetts, 1991.

[3] L. Jiang, V. Anantharam, and J. Walrand. How bad are selfish investments in network security? IEEE/ACM Transactions on Networking, 19(2):549-560, April 2011.

[4] P. Maillé, P. Reichl, and B. Tuffin. Interplay between security providers, consumers, and attackers: a weighted congestion game approach. In GameSec Second International Conference on Decision and Game Theory for Security, pages 67-86, College Park, MD, Maryland, United States, November 2011.

[5] P. Maillé, P. Reichl, and B. Tuffin. Of threats and costs: a game-theoretic approach to security risk management. In Performance Models and Risk Management in Communication Systems, Springer Optimization and Its Applications, pages 33-54. Springer, 2011.

[6] M. Osborne and A. Rubinstein. A Course in Game theory. MIT Press, 1994.

[7] J. B. Rosen. Existence and uniqueness of equilibrium points for concave $n$-person games. Econometrica, 33:520-534, 1965.

[8] G. Rubino. Network reliability evaluation. In K. Bagchi and J. Walrand, editors, State-of-the art in performance modeling and simulation. Gordon and Breach Books, 1998.

[9] C.K. Toh. Wireless ATM and Ad-Hoc Networks: Protocols and Architectures. Kluwer Academic Publisherb Group, 1997. 
[10] H. Varian. System reliability and free riding. In L. Jean Camp and Stephen Lewis, editors, Economics of Information Security, pages 1-15. Springer US, Boston, MA, 2004. 